\title{
DIGITALISIERUNGSPROJEKT ZU DEUTSCHSPRACHIGEN JÜ- DISCHEN ZEITUNGEN, ZEITSCHRIFTEN UND VOLKSKALENDERN AUS DEM ÖSTLICHEN EUROPA
}

von Albert Weber

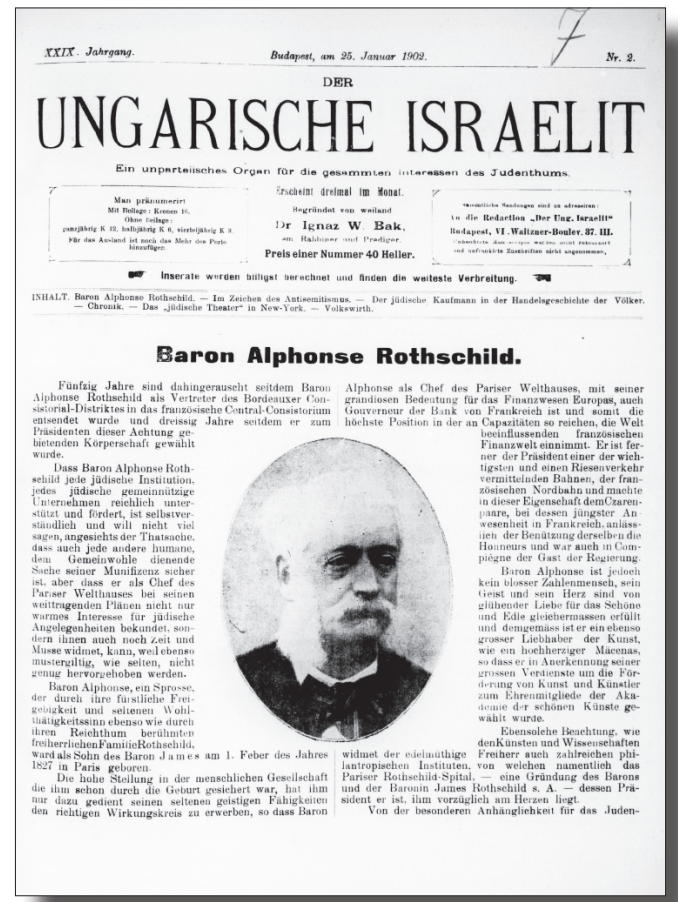

Abb. 1: Deutschsprachige jüdische Zeitungen aus Osteuropa - eine wichtige historische Quelle für bildliche Darstellungen und biographische Daten (ㄷ IOS Regensburg)

Zeitungen sind nicht nur "Sekundenzeiger der Geschichte“ (A. Schopenhauer), die als historische Quellen Tagespolitik und Wirtschaftsleben dokumentieren - sie sind auch Informationsträger zum Pluralitätsverständnis der Gesellschaften, in denen sie entstanden sind. Geradezu in Echtzeit zeigen sie die Debatten, Konflikte und Prozesse, die in anderen Medien und Materialgattungen oftmals nur partiell, selektiv oder stark verzerrt überliefert werden. Besonders sichtbar wird die gesellschaftliche Ausverhandlung von Toleranz, Assimilation oder Diskriminierung in der Presse ethnischer und religiöser Minderheiten: Die Presse initiiert und reflektiert Identitäts- 
und politische Diskurse, von denen Impulse ausgingen, die aufgrund des wachsenden Nationalismus im 19. und zu Beginn des 20. Jahrhunderts weitreichende Folgen für die europäischen Gesellschaften hatten. Die Zeitungen waren dabei ein wichtiges Forum der Arbeit einer Gemeinschaft an der eigenen Identität sowie der Bezugnahme zu anderen ethnischen Gruppen. Neben ideologischen Auseinandersetzungen hatten in Zeitungen verhandelte Themen somit Auswirkungen auf kulturelle Paradigmen und trugen zur Ausbildung von nationalen wie religiösen Identitäten bei. Zeitungen sind das Medium kollektiver Identitäts- und Gedächtnisbildung und stellen daher ein wissenschaftlich unverzichtbares und auch für die Öffentlichkeit ungemein interessantes Quellenmaterial für die Digitalisierung dar.

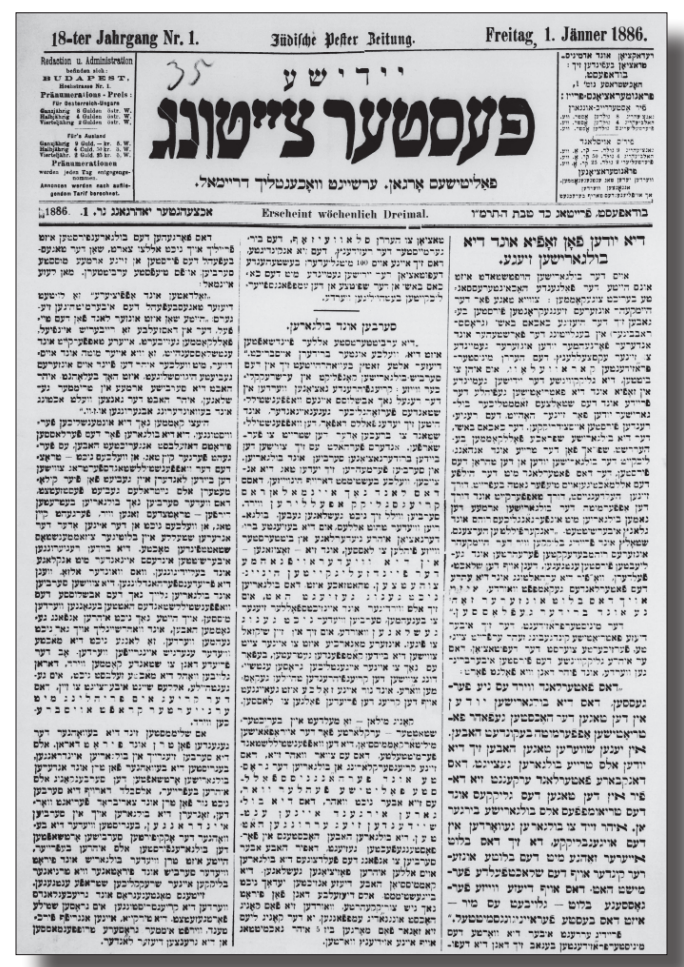

Abb. 2: Zeitzeuge einer gelebten Multikulturalität - die Jüdische Pester Zeitung aus Budapest in deutscher Sprache und hebräischer Schrift (๔ IOS Regensburg)

Die bisherigen Digitalisierungsprojekte der Bibliothek des Instituts für Ostund Südosteuropaforschung (IOS, Regensburg) haben ausschließlich die 
Periodika deutscher Minderheiten aus dem östlichen Europa berücksichtigt, so etwa der Ungarn-, Sudeten- und Wolgadeutschen, der Donauschwaben oder Siebenbürger Sachsen. ${ }^{1}$ Bezüge zu den jüdischen Bevölkerungen mit deutscher Muttersprache, die sich oftmals als Vertreter deutscher Kultur im östlichen Europa und somit als Deutsche jüdischen Glaubens verstanden, sind darin zwar vorhanden, jedoch nicht selten und insbesondere ab den 1930er-Jahren durch einen wachsenden Antisemitismus und eine damit einhergehende propagandistische Deformierung der Wahrnehmung jüdischen Lebens in Osteuropa geprägt. ${ }^{2}$ Als historische Quelle für Kultur und Geschichte der deutschsprachigen Juden können sie allerdings auch für frühere Jahrzehnte von der Forschung nur mit Einschränkungen rezipiert werden: Deutsche Zeitungen im östlichen Europa waren aufgrund ihres hohen Informationswertes zu verschiedenen gesellschaftlichen, kulturellen, politischen und wirtschaftlichen Anliegen freilich auch an eine jüdische Leserschaft adressiert, vertraten jedoch allgemein keine jüdischen Interessen und enthielten auch vor dem Ersten Weltkrieg bisweilen Artikel, die Stereotypen wiedergeben oder als offen antisemitisch kategorisiert werden können.

Die Digitalisierung jüdischer Periodika stellt deswegen sowie zum Zweck einer umfassenderen historischen Dokumentation der deutschsprachigen jüdischen Gemeinschaften Osteuropas ein wichtiges Desiderat dar. Hierfür hat das IOS mit Förderung der Bundesbeauftragten für Kultur und Medien aufgrund eines Beschlusses des Deutschen Bundestages in Zusammenarbeit mit der Universitätsbibliothek Regensburg sowie dem Digitalen Forum Mittel- und Osteuropa (DiFMOE, www.difmoe. eu) ein zwölfmonatiges Digitalisierungsprojekt initiiert: Ein umfassender Bestand historischer deutschsprachiger, teils in hebräischer Schrift gedruckter Zeitungen, Zeitschriften, Volkskalender, Jahrbücher und Almanache osteuropäischer Juden mit deutscher und jiddischer Muttersprache wurde ausgesucht, der nach der Digitalisierung (Mitte 2016) als volltextdurchsuchbares Quellenmaterial für Forschung, Lehre und Öffentlichkeit frei, uneingeschränkt und langzeitarchiviert auf dem DiFMOE zur Verfügung gestellt wird. ${ }^{3}$ Neben Originalvorlagen, die in Bibliotheken im Inund Ausland nachgewiesen sind, werden, insofern verfügbar, Mikrofilme eingescannt, womit eine materielle Beanspruchung der teils unikalen Originale vermieden wird. Im Anschluss an die Digitalisierung der Periodika werden auch bildliche Darstellungen wie etwa Zeichnungen, Abbildungen oder Fotografien separat in einem virtuellen Bildkatalog erfasst und über Schlagworte ausgezeichnet. Hierdurch soll neben den Textmaterialien auch ein übersichtlicher visueller Zugang zur Selbstdarstellung und zu den 
Identitätsoptionen historischer jüdischer Minderheiten im östlichen Europa gewährleistet werden.

Albert Weber, M.A. Institut für Ost- und Südosteuropaforschung Landshuter Straße 4, D-93047 Regensburg E-Mail:weber@ios-regensburg.de

$1 \mathrm{Vgl}$. http://www.ios-regensburg.de/informationsinfrastruktur/bibliothek/projekte/deutschsprachige-periodika.html.

$2 \mathrm{Vgl}$. exemplarisch folgende antisemitische Darstellungen: Siebenbürgisch-Deutsches Tageblatt vom 9. März 1941, S. 8: (http://www.difmoe.eu/ocr/page_view? active=Periodika\&name=Siebenb\%C3\%BCrgi sch-Deutsches+Tageblatt\&page_id=29092); Südböhmische Volkszeitung vom 14. November 1937, Bildbeilage S. 2 (http://www.difmoe. eu/ocr/page_view? active $=$ Periodika\&name $=S \% C 3 \% B C d b \% C 3 \% B 6 h m i s$ che+Volkszeitung\&page_id=1648).

3 Eine Auflistung und weitere Informationen zu den Periodika: http:// www.ios-regensburg.de/informationsinfrastruktur/bibliothek/projekte/juedische-deutschsprachige-periodika.html.

Dieses Werk ist lizenziert unter einer

Creative-Commons-Lizenz Namensnennung 4.0 International 